\title{
Rings in which elements are a sum of a central and a unit element
}

\author{
Yosum Kurtulmaz \\ Sait Halicioglu \\ Huanyin Chen
}

Abdullah Harmanci

\begin{abstract}
In this paper we introduce a new class of rings whose elements are a sum of a central and a unit element, namely a ring $R$ is called $C U$ if each element $a \in R$ has a decomposition $a=c+u$ where $c$ is central and $u$ is unit. One of the main results in this paper is that if $F$ is a field which is not isomorphic to $\mathbb{Z}_{2}$, then $M_{2}(F)$ is a $C U$ ring. This implies, in particular, that any square matrix over a field which is not isomorphic to $\mathbb{Z}_{2}$ is the sum of a central matrix and a unit matrix.
\end{abstract}

\section{Introduction}

Throughout this paper, all rings considered are associative with an identity unless otherwise stated. In what follows, $\mathbb{Z}, \mathbb{Q}$ and $\mathbb{C}$ denote the ring of integers, the ring of rational numbers and the ring of complex numbers, respectively. For a ring $R$, $U(R), J(R), N(R)$ and $C(R)$ denote the group of units, the Jacobson radical, the set of nilpotent elements and the center of $R$, respectively. For a positive integer $n, \mathbb{Z}_{n}$ is the ring of integers modulo $n$ and $M_{n}(R)$ denotes the full matrix ring over $R, U_{n}(R)$ is the subring of $M_{n}(R)$ consisting of all $n \times n$ upper triangular matrices and $G L_{n}(R)$ is the general linear group of $M_{n}(R)$.

If $A \in M_{n}(\mathbb{C})$, then there exists $c \in \mathbb{C}$ such that $c$ is not an eigenvalue of $A$. Hence, $A-c I_{n}$ is invertible in $M_{n}(\mathbb{C})$. Thus, $A=c I_{n}+\left(A-c I_{n}\right)$ is a

Received by the editors in November 2018 - In revised form in April 2019.

Communicated by S. Caenepeel.

2010 Mathematics Subject Classification : 15B33, 15B36, 16S70, 16 U99.

Key words and phrases : $\mathrm{CU}$ ring, $\mathrm{CU}$-decomposition, matrix ring, uniquely nil clean ring, *-clean ring, nil *-clean ring. 
$C U$-decomposition of $A$. Therefore $M_{n}(\mathbb{C})$ is a $C U$ ring. For more general case, let $F$ be an algebraically closed field. Then every element in $M_{n}(F)$ is a sum of a central and a unit element in $M_{n}(F)$. Motivated by these facts, we investigate elementary properties of rings in which the elements are the sum of a central and a unit element.

\section{Properties of $C U$ Rings}

In this section we introduce a $\mathrm{CU}$ ring and investigate its elementary properties. We give relations between $\mathrm{CU}$ rings and some related rings.

We now give our main definition.

Definition 2.1. Let $R$ be a ring. An element $a \in R$ has a $C U$-decomposition if $a=c+u$ for some $c \in C(R)$ and $u \in U(R)$. Then $R$ is called a $C U$ ring if every element of $R$ has a $C U$-decomposition.

Examples 2.2. (1) Every commutative ring is $\mathrm{CU}$.

(2) Every local ring is $C U$.

(3) Every nilpotent element in any ring has a $C U$-decomposition.

(4) $\mathrm{CU}$ property for rings is preserved under homomorphic images.

Proof. (1) If $a \in R$, then $a=1+(a-1)$.

(2) Let $a \in R$. Since $R$ is local, $a$ is invertible or $1-a$ is invertible. If $a$ is invertible, then $a=0+a$. On the other hand, we have $a=1+(a-1)$, if $1-a$ is invertible.

(3) Let $a$ be any nilpotent element in a ring $R$. Then $a$ has a CU-decomposition such that $a=1+(a-1)$.

(4) Clear since invertible elements and central elements are preserved under epimorphisms of rings.

Proposition 2.3. Let $R$ be a ring and $a \in R$. Then a has a $C U$-decomposition if and only if for each $p \in U(R)$, pap ${ }^{-1}$ has a CU-decomposition.

Proof. Assume that $a$ has a $C U$-decomposition. Then $a=c+u$ where $c$ is central and $u$ is invertible in $R$. For an invertible $p \in R$, $p a p^{-1}=p c p^{-1}+p u p^{-1}$ where $p c p^{-1}=c$ is central and $p u p^{-1}$ is invertible. Conversely, suppose that $p a p^{-1} \in R$ has a $C U$-decomposition. So $p^{-1} p^{-1}=t+x$ where $t$ is central and $x$ is invertible. Hence $a=p^{-1} t p+p^{-1} x p$ is a CU-decomposition of $a$.

Lemma 2.4. Let $R$ be a commutative ring. For any positive integer $n, A \in M_{n}(R)$ has a CU-decomposition if and only if $A-c I_{n} \in G L_{n}(R)$ for some $c \in R$.

Proof. Assume that $A \in M_{n}(R)$ has a $C U$-decomposition. By assumption there exists $c \in R$ such that $A-c I_{n} \in G L_{n}(R)$. Note that for any $B \in M_{n}(R), B$ is central in $M_{n}(R)$ if and only if there exists $c \in R$ such that $B=c I_{n}$. Conversely, suppose that for any $A \in M_{n}(R)$, there exists $c \in R$ such that $A-c I_{n} \in G L_{n}(R)$. As $c I_{n}$ is central in $M_{n}(R), A \in M_{n}(R)$ has a $C U$-decomposition.

For a commutative ring $R$, the following result is important to find out whether $A \in M_{n}(R)$ has a $C U$-decomposition. 
Corollary 2.5. Let $R$ be a commutative ring. For any positive integer $n, A \in M_{n}(R)$ has a CU-decomposition if and only if $f(c)$ is invertible in $R$ for some $c \in R$, where $f(x) \in R[x]$ is the characteristic polynomial of $A$.

Proof. Note that in a commutative ring $R$, for any $A \in M_{n}(R)$ and $c \in R$, $A-c I_{n} \in G L_{n}(R)$ if and only if $f(c)=\operatorname{det}\left(A-c I_{n}\right)$ is invertible in $R$. The result is clear by Lemma 2.4.

For a positive integer $n$, one may suspect that if $R$ is a $C U$ ring, then the full matrix ring $M_{n}(R)$ is $C U$. The following example shows that this is not true in general.

Example 2.6. Since $\mathbb{Z}$ is commutative, it is a $C U$ ring. But $M_{2}(\mathbb{Z})$ is not a $C U$ ring. Consider $A=\left[\begin{array}{ll}1 & 2 \\ 3 & 6\end{array}\right] \in M_{2}(\mathbb{Z})$ which is not invertible. Let $f(\lambda)=$ $\operatorname{det}\left(A-\lambda I_{2}\right)=\lambda(\lambda-7)$ denote the characteristic polynomial of $A$. Then there is no $c \in \mathbb{Z}$ such that $f(c)$ is invertible in $\mathbb{Z}$. Hence $A$ has not a $C U$-decomposition by Corollary 2.5 .

Theorem 2.7. Let $R$ be a division ring. For any positive integer $n, M_{n}(R)$ is a CU ring if and only if $|C(R)|>n$.

Proof. Assume that $|C(R)| \leq n$. Consider $A$ as a diagonal matrix which has the property that each element of $C(R)$ is one of the diagonal entries of $A$. For such a matrix $A$ there is no $c \in C(R)$ for which $A-c I$ is a unit. Hence $M_{n}(R)$ is not a CU ring. For the reverse implication, we will prove something a little stronger. We show that if $|C(R)|>n$, then for every $A \in M_{n}(R)$, there are at most $n$ elements $c \in C(R)$ for which $A-c I$ is not a unit. We complete the proof by induction on $n$. If $n=1$, the result is clear. Suppose that for every $0<m<n$ and $A \in M_{m}(R)$, there exist at most $m$ elements $c \in C(R)$ for which $A-c I$ is not a unit. Let $A \in M_{n}(R)$ and $c \in C(R)$. If $A-c I$ is a unit or nilpotent, we are done. Otherwise, $A-c I$ is neither a unit nor nilpotent. By Fitting's Lemma, applied to $A-c I$, we know that $A-c I$ is similar to a block diagonal matrix $\operatorname{diag}\left(A_{1}, A_{2}\right)$, where $A_{1}$ is a unit and $A_{2}$ is nilpotent and $A_{1} \in M_{m}(R)$ and $A_{2} \in M_{n-m}(R)$, where $0<m<n$, since $A-c I$ is neither a unit nor is nilpotent. Since $c$ is central, $A$ is similar to $\operatorname{diag}\left(B_{1}, B_{2}\right)$ where $B_{1}=A_{1}+c I$ and $B_{2}=A_{2}+c I$. By induction, there are at most $m$ elements $c \in C(R)$ for which $B_{1}-c I$ is not a unit, and at most $n-m$ elements $c \in C(R)$ for which $B_{2}-c I$ is not a unit. It follows that there are at most $n=m+(n-m)$ elements $c \in C(R)$ for which $A-c I$ is not a unit.

The following is more a corollary of the above proof, rather than the statement, the center of $R$ need not map onto the center of $R / J(R)$, even for local rings, namely, skew power series rings. 
Corollary 2.8. Let $R$ be local ring. For a positive integer $n, M_{n}(R)$ is a CU ring if and only if the image of $C(R)$ in $R / J(R)$ has strictly more than $n$ elements.

Proof. Note that $R / J(R)$ is a division ring, and $a \in R$ is invertible in $R$ if and only the image of $a$ in $R / J(R)$ is invertible in $R / J(R)$. So the forward implication is clear. For the reverse implication, let $A \in M_{n}(R)$. We know that there are at most $n$ elements in $C(R / J(R))$ for which the image of $A-c I$ is not a unit in $M_{n}(R / J(R))$. Since $M_{n}(R / J(R)) \cong M_{n}(R) /\left(M_{n}(J(R)) \cong M_{n}(R) / J\left(M_{n}(R)\right)\right.$, $A-c I$ is not a unit in $M_{n}(R)$. Since the image of $C(R)$ in $C(R / J(R))$ has strictly more than $n$ elements, there exists at least one $c \in C(R)$ for which $A-c I$ is unit.

Theorem 2.9. Let $R$ be a commutative local ring. For any positive integer $n, M_{n}(R)$ is a CU ring if and only if $R / J(R)$ is not isomorphic to $\mathbb{Z}_{2}$.

Proof. Assume that $M_{n}(R)$ is a $C U$ ring. By contradiction, suppose that $R / J(R)$ is isomorphic to $\mathbb{Z}_{2}$. Let $A=\left[\begin{array}{ll}1 & 1 \\ 0 & 0\end{array}\right] \in M_{2}(R)$ and $B=A \oplus 0_{n-2} \in M_{n}(R)$ where $0_{n-2}$ is the $(n-2) \times(n-2)$ zero matrix and $f(\lambda)=\operatorname{det}\left(B-\lambda I_{n}\right)$ is the characteristic polynomial of $B$. By Corollary 2.5, there exists $c \in R$ such that $f(c)=c^{n-1}(c-1)$ is invertible in $R$. Hence $c$ is nonzero, therefore $c \in J(R)$ or $c-1 \in J(R)$. This is a contradiction. Conversely, since $R / J(R)$ is a field and $M_{n}(R) / J\left(M_{n}(R)\right) \cong M_{n}(R / J(R))$, we may assume that $R$ is a field and $R$ is not isomorphic to $\mathbb{Z}_{2}$. Let $A \in M_{n}(R)$. If $A \in G L_{n}(R)$, then there is nothing to do. Assume that $A \notin G L_{n}(R)$. We complete the proof by induction on $n$. Assume that $n=2$ and $A=\left[\begin{array}{ll}a & b \\ c & d\end{array}\right]$. Since $A$ is not invertible, $a d=b c$. Being $R \nsucceq \mathbb{Z}_{2}$, there exists $0 \neq u \in R$ such that $a+d-u \neq 0$. Let $C=u I_{2}$. Then $C$ is central and $A-C \in G L_{2}(R)$ since $\operatorname{det}(A-C)=-u(a+d-u) \neq 0$.

Assume that the claim holds for all $k<n$ and $A \in M_{n}(R)$. Since $R$ is a local ring, it follows by [1, Corollary 7.3.2] that there exist $P, Q \in G L_{n}(R)$ such that $P A Q=\operatorname{diag}\left(I_{r}, 0_{n-r}\right)$. We have

$$
P A P^{-1}=\operatorname{diag}\left(I_{r}, 0_{n-r}\right) Q^{-1} P^{-1}=\left(\begin{array}{cc}
A_{1} & A_{2} \\
0 & 0
\end{array}\right) .
$$

By induction hypothesis, there exist nonzero $c \in R, U \in G L_{n}(R)$ such that $A_{1}=\operatorname{diag}(c, c, \ldots, c)+U$. Then

$$
P A P^{-1}=\operatorname{diag}(c, c, \ldots, c)+\left[\begin{array}{cc}
U & A_{2} \\
0 & \operatorname{diag}(-c,-c, \ldots,-c)
\end{array}\right] .
$$

By Proposition 2.3, $A$ has a $C U$-decomposition.

Corollary 2.10. Let $F$ be a field. For any positive integer $n, M_{n}(F)$ is $C U$ if and only if $F$ is not isomorphic to $\mathbb{Z}_{2}$.

Let $R$ be a ring and $U_{n}(R)$ the subring of $M_{n}(R)$ consisting of all $n \times n$ upper triangular matrices. Then we have the following. 
Corollary 2.11. Let $R$ be a commutative ring. For a positive integer $n, A \in U_{n}(R)$ has a CU-decomposition if and only if there exists $c \in R$ such that $A-c I_{n}$ is invertible in $U_{n}(R)$.

Proof. Same as the proof of Lemma 2.4.

The following result can be useful to determine under what conditions the ring of $2 \times 2$ upper triangular matrices $U_{2}(R)$ is $C U$.

Proposition 2.12. Let $R$ be a commutative ring. Then $U_{2}(R)$ is a $C U$ ring if and only if for any $a, b \in R$, there exists $c \in R$ such that $a-c, b-c \in U(R)$.

Proof. Assume that $U_{2}(R)$ is $C U$. Let $a, b \in R$. Consider $A=\left[\begin{array}{ll}a & 0 \\ 0 & b\end{array}\right] \in U_{2}(R)$. By Corollary 2.11, there exists $c \in R$ such that $A-c I_{2}$ is invertible in $U_{2}(R)$. Hence $a-c$ and $b-c$ are invertible. Conversely, let $A=\left[\begin{array}{ll}x & y \\ 0 & z\end{array}\right] \in U_{2}(R)$. There exists $c \in C(R)$ such that $x-c, z-c \in U(R)$. Let $U=\left[\begin{array}{cc}x-c & y \\ 0 & z-c\end{array}\right]$ and $C=\left[\begin{array}{ll}c & 0 \\ 0 & c\end{array}\right]$. Then $C \in C\left(U_{2}(R)\right)$ and $U \in U\left(U_{2}(R)\right)$. Hence $A=C+U$ is a $C U$-decomposition of $A$.

For a positive integer $n$, the next example shows that if $R$ is a $C U$ ring, then $U_{n}(R)$ need not be a $C U$ ring.

Example 2.13. Consider the ring $\mathbb{Z}$, let $a=2$ and $b=3$. Then there is no $c \in \mathbb{Z}$ such that $2-c$ and $3-c$ are invertible. By Proposition $2.12, U_{2}(\mathbb{Z})$ is not $C U$.

In spite of the fact that $U_{n}(R)$ need not be a $C U$ ring for any positive integer $n$ and for some rings $R$, we now show that $C U$ subrings of $U_{n}(R)$ are rich. If $D_{n}(R)=\left\{\left(a_{i j}\right) \in U_{n}(R) \mid\right.$ all diagonal entries of $\left(a_{i j}\right)$ are equal $\}$, then we have the following result.

Proposition 2.14. Let $R$ be a ring. For any positive integer $n, R$ is a CU ring if and only if $D_{n}(R)$ is a CU ring.

Proof. We assume that $R$ is a $C U$ ring and $a \in R$. Let $A=\operatorname{diag}(a, a, \ldots, a)+\left(a_{i j}\right) \in$ $D_{n}(R)$ with $i<j$. Then $a$ has $C U$-decomposition such as $a=c+u$ where $c$ is central and $u$ is invertible. Consider $C=\operatorname{diag}(c, c, \ldots, c)$ and $U=\operatorname{diag}(u, u, \ldots, u)+\left(a_{i j}\right)$ with $i<j$. Then $C$ is central and $U$ is invertible in $D_{n}(R)$. Hence $A=C+U$ is a $C U$-decomposition of $A$. Conversely, suppose that $D_{n}(R)$ is a $C U$ ring for some positive integer $n$. Let $a \in R$ and consider $A=\operatorname{diag}(a, a, \ldots, a) \in D_{n}(R)$. Then there exist central $C=\operatorname{diag}(c, c, \ldots, c) \in$ $D_{n}(R)$ and invertible $U=\operatorname{diag}(u, u, \ldots, u) \in D_{n}(R)$ such that $A=C+U$. Hence $a=c+u$ is the CU-decomposition of $a$. 
Let $V_{n}(R)$ the subrings of $U_{n}(R)$ where $n$ is a positive integer.

$$
V_{n}(R)=\left\{\left[\begin{array}{cccccc}
a_{1} & a_{2} & a_{3} & \ldots & a_{n-1} & a_{n} \\
0 & a_{1} & a_{2} & \ldots & a_{n-2} & a_{n-1} \\
0 & 0 & a_{1} & \ldots & a_{n-3} & a_{n-2} \\
\vdots & \vdots & \vdots & \ddots & \vdots & \vdots \\
0 & 0 & 0 & \ldots & a_{1} & a_{2} \\
0 & 0 & 0 & \ldots & 0 & a_{1}
\end{array}\right] \mid a_{i} \in R, 1 \leq i \leq n\right\}
$$

Let $\left(x^{n}\right)$ denote the ideal generated by $x^{n}$ in $R[x]$. Then we have $R[x] /\left(x^{n}\right) \cong$ $V_{n}(R)$ in a natural way.

Theorem 2.15. Let $R$ be a ring. For any positive integer $n$, the following statements are equivalent.

(1) $R$ is a CU ring.

(2) $V_{n}(R)$ is a CU ring.

Proof. (2) $\Rightarrow(1)$ Let $r \in R$ and $A=\left\{\left(a_{i}\right) \mid a_{1}=r\right.$ and $a_{i}=0$ if $\left.i \neq 1\right\} \in V_{n}(R)$. By (2) $A$ has a $C U$-decomposition $A=C+U$ where $U=\left(u_{i}\right)$ is invertible and $C=\left(c_{i}\right)$ central in $V_{n}(R)$. Then $u_{1}$ is invertible in $R$ and $c_{1}$ is central in $R$. Hence $r=c_{1}+u_{1}$ is a $C U$-decomposition of $r$ in $R$.

$(1) \Rightarrow(2)$ Let $A=\left(a_{i}\right)=\left[\begin{array}{cccccc}a_{1} & a_{2} & a_{3} & \ldots & a_{n-1} & a_{n} \\ 0 & a_{1} & a_{2} & \ldots & a_{n-2} & a_{n-1} \\ 0 & 0 & a_{1} & \ldots & a_{n-3} & a_{n-2} \\ \vdots & \vdots & \vdots & \ddots & \vdots & \vdots \\ 0 & 0 & 0 & \ldots & a_{1} & a_{2} \\ 0 & 0 & 0 & \ldots & 0 & a_{1}\end{array}\right] \in V_{n}(R)$. There are invertible elements $u_{i}$ in $R$ and central elements $c_{i}$ of $R$ with $a_{i}=c_{i}+u_{i}$. Let

$$
\begin{aligned}
U=\left(u_{i}\right)= & {\left[\begin{array}{cccccc}
u_{1} & u_{2} & u_{3} & \ldots & u_{n-1} & u_{n} \\
0 & u_{1} & u_{2} & \ldots & u_{n-2} & u_{n-1} \\
0 & 0 & u_{1} & \ldots & u_{n-3} & u_{n-2} \\
\vdots & \vdots & \vdots & \ddots & \vdots & \vdots \\
0 & 0 & 0 & \ldots & u_{1} & u_{2} \\
0 & 0 & 0 & \ldots & 0 & u_{1}
\end{array}\right] \in V_{n}(R), } \\
C=\left(c_{i}\right)= & {\left[\begin{array}{cccccc}
c_{1} & c_{2} & c_{3} & \ldots & c_{n-1} & c_{n} \\
0 & c_{1} & c_{2} & \ldots & c_{n-2} & c_{n-1} \\
0 & 0 & c_{1} & \ldots & c_{n-3} & c_{n-2} \\
\vdots & \vdots & \vdots & \ddots & \vdots & \vdots \\
0 & 0 & 0 & \ldots & c_{1} & c_{2} \\
0 & 0 & 0 & \ldots & 0 & c_{1}
\end{array}\right] \in V_{n}(R) . }
\end{aligned}
$$

Then $A=C+U$ is a $C U$-decomposition of $A$. 
Lemma 2.16. Let $R$ be a ring and e an idempotent in $R$. Then we have the following.

(1) If $u \in U(R)$ and $e u=u e$, then eue is invertible in eRe.

(2) If $c \in C(R)$, then ec is central in eRe.

Proof. (1) Let $u u^{-1}=1=u^{-1} u$ and $e u=u e$. Then $e u^{-1}=u^{-1} e$. Hence $e=e u^{-1}=(e u e)\left(e u^{-1} e\right)$. Similarly, $e=e u^{-1} u=\left(e u^{-1} e\right)(e u e)$. So $e u^{-1} e$ is the inverse of eue in $e$ Re.

(2) Let exe $\in e R e$. Note that $e c=e c e=c e$. Then $($ exe $)(e c)=($ exe $) c=c($ exe $)=$ $(c e)(e x e)$. Hence $e c$ is central in $e$ Re.

Theorem 2.17. Let $R$ be a $C U$ ring and $e^{2}=e \in R$. Then the corner ring $e R e$ is $C U$.

Proof. Let eae $\in e R e$. By assumption eae $=u+c$, for some $u \in U(R)$ and $c \in C(R)$. Then eae $-u$ is central. By commuting eae $-u$ with $e$, we have $e u=u e$. By Lemma 2.16, eue is invertible in $e$ Re and ece is central in $e R e$. Hence $e a e=e c e+e u e$ is the CU-decomposition of eae in $e R e$. Thus $e R e$ is $C U$.

As a direct consequence of Theorem 2.17, we have the following.

Proposition 2.18. Let $R$ be a ring. For any positive integer $n$, if $M_{n}(R)$ is a $C U$ ring, then $R$ is a CU ring.

Proof. Let $n$ be any positive integer and $e_{11} \in M_{n}(R)$ denote the $n \times n$ matrix unit with $(1,1)$ entry 1 elsewhere 0 . By Theorem $2.17, e_{11} M_{n}(R) e_{11}$ is a $C U$ ring. Since $e_{11} M_{n}(R) e_{11}$ is isomorphic to $R, R$ is a $C U$ ring.

The next result shows that being $\mathrm{CU}$ for rings is preserved under the direct products of rings.

Proposition 2.19. Let $R=\prod_{i \in I} R_{i}$ be a direct product of rings. Then $R$ is a $C U$ ring if and only if $R_{i}$ is a CU ring for each $i \in I$.

Proof. We may assume that $I=\{1,2\}$ and $R=R_{1} \times R_{2}$. Note that $C(R)=C\left(R_{1}\right) \times C\left(R_{2}\right)$ and $U(R)=U\left(R_{1}\right) \times U\left(R_{2}\right)$. For the necessity, let $r_{1} \in R_{1}$. Then $\left(r_{1}, 0\right)=\left(c_{1}, c_{2}\right)+\left(u_{1}, u_{2}\right)$ where $\left(u_{1}, u_{2}\right)$ is invertible in $R$ and $\left(c_{1}, c_{2}\right)$ is central in $R$. Hence $r_{1}=c_{1}+u_{1}$ is a CU-decomposition of $r_{1} \in R_{1}$. So $R_{1}$ is a $C U$ ring. A similar proof takes care for $R_{2}$ be $C U$. For the sufficiency, assume that $R_{1}$ and $R_{2}$ are $C U$. Let $\left(r_{1}, r_{2}\right) \in R$. By assumption $r_{1}$ and $r_{2}$ have CU-decompositions $r_{1}=c_{1}+u_{1}$ and $r_{2}=c_{2}+u_{2}$ where $u_{1}$ is invertible in $R_{1}$, $c_{1}$ is central in $R_{1}$ and $u_{2}$ is invertible in $R_{2}, c_{2}$ is central in $R_{2}$. Hence $\left(r_{1}, r_{2}\right)$ has a $C U$-decomposition $\left(r_{1}, r_{2}\right)=\left(c_{1}, c_{2}\right)+\left(u_{1}, u_{2}\right)$. The same proof works for any index set $I$.

Recall that a ring $R$ is called unit-central [4] if all unit elements are central in $R$.

Lemma 2.20. Every unit-central $\mathrm{CU}$ ring is commutative.

Proof. Assume that $R$ is a unit-central $C U$ ring. Let $a \in R$ with $a=c+u$ where $c$ is central and $u$ is unit. By assumption $u$ is central. So $a$ is central. 
Recall that in [2], uniquely nil clean rings are defined. An element $a$ in a ring $R$ is called uniquely nil clean if there is a unique idempotent $e$ such that $a-e$ is nilpotent. The ring $R$ is uniquely nil clean if each of its elements is uniquely nil clean. It is proved that in a uniquely nil clean ring every idempotent is central [2, Lemma 5.5]. In fact, if $e$ is an idempotent in a uniquely clean ring $R$, for any $r \in R$, then $e+(r e-e r e)$ can be written in two ways as a sum of an idempotent and a nilpotent as $e+($ re - ere $)=(e+($ re - ere $))+0=e+($ re - ere $)$. Then $e=e+(r e-e r e)$ and $r e-e r e=0$. Similarly, $e r-e r e=0$. Hence $e$ is central. Let $R$ be a ring with involution $*$. In [7], a ring is called ${ }^{*}$-clean if each of its elements is a sum of a unit and a projection, and $R$ is strongly *-clean if each of its elements is a sum of a unit and a projection that commute with each other. It is proved that every strongly *-clean ring is abelian in [7, Lemma 2.1]. In [3], strongly nil *-clean rings are investigated. A ring is called strongly nil *-clean if every element of $R$ is the sum of a projection and a nilpotent that commute with each other.

Proposition 2.21. (1) Every uniquely nil clean ring is $\mathrm{CU}$.

(2) Every strongly *-clean ring is $\mathrm{CU}$.

(3) Every strongly nil *-clean ring is $\mathrm{CU}$.

Proof. (1) Let $R$ be a uniquely nil clean ring and $a \in R$. Then there exists a unique idempotent $e$ such that $(a+1)-e=b$ is nilpotent. Then $a=e+(b-1)$. By hypothesis $e$ is central and $b-1$ is invertible. Hence $R$ is a $C U$ ring.

(2) Assume that $R$ is a strongly ${ }^{*}$-clean ring. Let $a \in R$ with $a=u+p$ where $u$ is unit and $p$ is a projection with $u p=p u$. Since $R$ is abelian, $p$ is central in $R$. Thus $a=u+p$ is a $C U$-decomposition of $a$.

(3) By [3, Proposition 2.5], every strongly nil *-clean ring is strongly *-clean ring. By (2), if $R$ is a strongly nil *-clean ring, then it is a $C U$ ring.

In Example 3.6, we show that $C U$ rings need not be uniquely nil clean.

\section{Extensions of $C U$ rings}

In this section, we study some extensions of $C U$ rings. In particular, we investigate under what conditions the Dorroh extension of $R$, the formal triangular matrix ring and some subrings of the ring of all $n \times n$ matrices $M_{n}(R)$ are $C U$.

Let $R$ be a ring and $D(\mathbb{Z}, R)$ denote the Dorroh extension of $R$ by the ring of integers $\mathbb{Z}$. Then $D(\mathbb{Z}, R)$ is the ring defined by the direct sum $\mathbb{Z} \oplus R$ with componentwise addition and multiplication $(n, r)(m, s)=(n m, n s+m r+r s)$ where $(n, r),(m, s) \in D(\mathbb{Z}, R)$. It is clear that $C(D(\mathbb{Z}, R))=\mathbb{Z} \oplus C(R)$. The identity of $D(\mathbb{Z}, R)$ is $(1,0)$ and the set of invertible elements is

$$
U(D(\mathbb{Z}, R))=\{(1, u) \mid u+1 \in U(R)\} \cup\{(-1, u) \mid u-1 \in U(R)\} .
$$

Theorem 3.1. Let $R$ be a ring. Then $R$ is a $C U$ ring if and only if $D(\mathbb{Z}, R)$ is $C U$.

Proof. Assume that $R$ is a $C U$ ring. Let $(n, r) \in D(\mathbb{Z}, R)$. Since $R$ is $C U, r=c+u$ where $u \in U(R)$ and $c \in C(R)$. Then $(n, r)=(n-1, c+1)+(1, u-1)$ is a $C U$-decomposition of $(n, r)$. Conversely, let $r \in R$. Then $(0, r)=(-1, c)+(1, u)$ or $(0, r)=(1, c)+(-1, u)$. Hence $r=(c-1)+(u+1)$ or $r=(c+1)+(u-1)$. So $R$ is $C U$. 
Let $R$ be a ring and $S$ a subring of $R$ and

$$
T[R, S]=\left\{\left(r_{1}, r_{2}, \cdots, r_{n}, s, s, \cdots\right): r_{i} \in R, s \in S, n \geq 1,1 \leq i \leq n\right\} .
$$

Then $T[R, S]$ is a ring under the componentwise addition and multiplication. Note that $U(T[R, S])=T[U(R), U(R) \cap U(S)]$ and $C(T,[R, S])=T[C(R)$, $C(R) \cap C(S)]$.

Proposition 3.2. Let $R$ be a ring and $S$ a subring of $R$. Then the following are equivalent.

1. $T[R, S]$ is a $C U$ ring.

2. $R$ and $S$ are $C U$.

Proof. (1) $\Rightarrow(2)$ Assume that $T[R, S]$ is $C U$ and let $a \in R$. Consider $X=(a, 0,0, \cdots) \in T[R, S]$. From the assumption there exist an invertible element $U=\left(u_{1}, u_{2}, \cdots, u_{m}, t, t, \cdots\right)$ and a central element $C=\left(c_{1}, c_{2}, \cdots, c_{n}, s, s, \cdots\right)$ in $T[R, S]$ such that $X=C+U$. By this equality $a=c_{1}+u_{1}$ is $C U$-decomposition of $a$. To see $S$ is a $C U$ ring, let $s \in S$. Then, $A=(0,0, \cdots, 0, s, s, \cdots) \in T[R, S]$. Since $T[R, S]$ is $C U$, we have $A=C+U$ where $U=\left(u_{1}, u_{2}, \cdots, u_{m}, v, v, \cdots\right)$ is invertible and $C=\left(c_{1}, c_{2}, \cdots, c_{m}, w, w, \cdots\right)$ is central. Hence, $s=w+v$ is a $C U$-decomposition of $s$.

(2) $\Rightarrow(1)$ Let $R$ and $S$ be $C U$ rings and $Y=\left(a_{1}, a_{2}, \cdots, a_{n}, s, s, s, \cdots\right)$ be an arbitrary element in $T[R, S]$. Then there exist $c_{i} \in C(R), u_{i} \in U(R), c \in C(R) \cap C(S)$ and $u \in U(R) \cap U(S)$ where $1 \leq i \leq n$, such that $a_{i}=c_{i}+u_{i}$ for all $1 \leq i \leq n$ and $s=c+u$. Then $Y=\left(u_{1}, u_{2}, \cdots, u_{n}, u, u, \cdots\right)+\left(c_{1}, c_{2}, \cdots, c_{n}, c, c, \cdots\right)$ is a $C U$ decomposition of $Y$ in $T[R, S]$.

In the sequel, we investigate under what conditions subrings of $M_{n}(R)$ are $\mathrm{CU}$ rings.

The rings $H_{(s, t)}(R):$ Let $R$ be a ring, and let $s, t \in C(R)$. Let

$$
H_{(s, t)}(R)=\left\{\left[\begin{array}{lll}
a & 0 & 0 \\
c & d & e \\
0 & 0 & f
\end{array}\right] \in M_{3}(R) \mid a, c, d, e, f \in R, a-d=s c, d-f=t e\right\} .
$$

Then $H_{(s, t)}(R)$ is a subring of $M_{3}(R)$. Note that $A=\left[\begin{array}{lll}a & 0 & 0 \\ c & d & e \\ 0 & 0 & f\end{array}\right] \in H_{(s, t)}(R)$ if and only if $A \in M_{3}(R)$ and $a-d=s c, d-f=t e$ if and only if $A=\left[\begin{array}{ccc}s c+t e+f & 0 & 0 \\ c & t e+f & e \\ 0 & 0 & f\end{array}\right]$. 
Lemma 3.3. Let $R$ be a ring, and let $s, t \in C(R)$. Then

$C\left(H_{(s, t)}(R)\right)=\left\{\left[\begin{array}{ccc}s c+t e+f & 0 & 0 \\ c & t e+f & e \\ 0 & 0 & f\end{array}\right] \in H_{(s, t)}(R) \mid c, e, f \in C(R)\right\}$.

Proof. Let $A=\left[\begin{array}{ccc}s c+t e+f & 0 & 0 \\ c & t e+f & e \\ 0 & 0 & f\end{array}\right] \in C\left(H_{(s, t)}(R)\right)$. Let $x \in R$ and $B=x e_{11}+x e_{22}+x e_{33} \in H_{(s, t)}(R)$ where $e_{i j}$ denote the matrix units in $M_{3}(R)$. Then $A B=B A$ implies, among others, $c x=x c$, ex $=x e$ and $f x=x f$. Since $s$ and $t$ are central, all components of $A$ are central.

Conversely, let $A=\left[\begin{array}{ccc}s c+t e+f & 0 & 0 \\ c & t e+f & e \\ 0 & 0 & f\end{array}\right] \in H_{(s, t)}(R)$ with $c, e$ and $f$ of $A$ are central. Let $B=\left[\begin{array}{ccc}s y+t u+v & 0 & 0 \\ y & t u+v & u \\ 0 & 0 & v\end{array}\right] \in H_{(s, t)}(R)$. We show that $A B=B A$. In fact $(3,3)$ component of $A B$ is $f v=v f$ is the $(3,3)$ component of $B A$ since $f$ is central $R$. $(2,3)$ component of $A B$ is $(t e+f) u+e v$. Since $f u=u f$ and $e$ is central, $(t e+f) u=u(t e+f)$. Hence $(t e+f) u+e v$ is the $(2,3)$ component of $B A . \quad(2,2)$ component of $A B$ is $(t e+f)(t u+v)$. Since te $+f$ is central and $(t e+f)(t u+v)=(t u+v)(t e+f),(t u+v)(t e+f)$ is the $(2,2)$ component of $B A . \quad(2,1)$ component of $A B$ is $c(s y+t u+v)+(t e+f) y$, and then $c(s y+t u+v)+(t e+f) y=y(s c+t e+f)+(t u+v) c$ is $(2,1)$ component of $B A$. $(1,1)$ component of $A B$ is $(s c+t e+f)(s y+t u+v)$. Since $s c+t e+f$ is central in $R,(s c+t e+f)(s y+t u+v)=(s y+t u+v)(s c+t e+f)$ is the $(1,1)$ component of $B A$. Hence $A B=B A$ for all $B \in H_{(s, t)}(R)$. Thus $A$ is central in $H_{(s, t)}(R)$.

Lemma 3.4. Let $R$ be a ring, and let $s, t \in C(R)$. Then the set of all invertible elements of $H_{(s, t)}(R)$ is

$$
U\left(H_{(s, t)}(R)\right)=\left\{\left[\begin{array}{lll}
a & 0 & 0 \\
c & d & e \\
0 & 0 & f
\end{array}\right] \in H_{(s, t)}(R) \mid a, d, f \in U(R), c, e \in R\right\} .
$$

Proof. Assume that $a, d$ and $f$ are invertible and let $a^{-1}, d^{-1}$ and $f^{-1}$ denote the inverses of $a, d$ and $f$ respectively. Let $A=\left[\begin{array}{lll}a & 0 & 0 \\ c & d & e \\ 0 & 0 & f\end{array}\right] \in H_{(s, t)}(R)$ and $B=$ $\left[\begin{array}{ccc}a^{-1} & 0 & 0 \\ -d^{-1} c a^{-1} & d^{-1} & -d^{-1} e f^{-1} \\ 0 & 0 & f^{-1}\end{array}\right] \in H_{(s, t)}(R)$. Then $A B=B A=I_{n}$. Since $a-d=s c$ if and only if $a^{-1}-d^{-1}=-s d^{-1} c a^{-1}$ and $d-f=t e$ if and only if $d^{-1}-f^{-1}=$ $-t d^{-1} e f^{-1}, B=A^{-1} \in H_{(s, t)}(R)$.

Conversely, suppose that $A=\left[\begin{array}{lll}a & 0 & 0 \\ c & d & e \\ 0 & 0 & f\end{array}\right] \in H_{(s, t)}(R)$ is invertible in $H_{(s, t)}(R)$ 
with inverse $B=\left[\begin{array}{ccc}x & 0 & 0 \\ y & z & u \\ 0 & 0 & v\end{array}\right]$. Then $A B=B A=I_{n}$. Comparing entries we reach $a x=x a=1, d z=z d=1$ and $f v=v f=1$. Hence $a, d$ and $f$ are invertible.

Theorem 3.5. Let $R$ be a ring, and let $s, t \in C(R) \cap J(R)$. Then $R$ is a CU ring if and only if $H_{(s, t)}(R)$ is $C U$.

Proof. Assume that $R$ is $C U$. Let $A=\left[\begin{array}{lll}a & 0 & 0 \\ c & d & e \\ 0 & 0 & f\end{array}\right] \in H_{(s, t)}(R)$. Let $f=f_{1}+f_{2}$, $e=e_{1}+e_{2}$ and $c=c_{1}+c_{2}$ denote the $C U$-decompositions of $f, e$ and $c$ where $f_{1}, e_{1}, c_{1} \in U(R)$ and $f_{2}, e_{2}, c_{2} \in C(R)$. Choose $d_{2}=f_{2}+t e_{2}$ and $a_{2}=d_{2}+s c_{2}$. By Lemma 3.3, $C=\left[\begin{array}{ccc}a_{2} & 0 & 0 \\ c_{2} & d_{2} & e_{2} \\ 0 & 0 & f_{2}\end{array}\right] \in C\left(H_{(s, t)}(R)\right)$. Moreover, $d-d_{2}=d-f_{2}-$ $t e_{2}=d-f+f_{1}-t e_{2}=f_{1}-t e-t e_{2}$ is invertible since $f_{1} \in U(R)$ and $t e-t e_{2} \in$ $J(R)$. Similarly $a-a_{2}=d-d_{2}+s c-s c_{2} \in U(R)$. Let $U=\left[\begin{array}{ccc}a_{1} & 0 & 0 \\ c_{1} & d_{1} & e_{1} \\ 0 & 0 & f_{1}\end{array}\right]$ with $a_{1}=a-a_{2}$ and $d_{1}=d-d_{2}$. By Lemma 3.4, $U \in U\left(H_{(s, t)}(R)\right)$. Hence $A=C+U$ is a $C U$-decomposition of $A$.

Conversely, suppose that $H_{(s, t)}(R)$ is $C U$ and $a \in R$.

Let $A=\left[\begin{array}{ccc}a & 0 & 0 \\ 0 & a & 0 \\ 0 & 0 & a\end{array}\right] \in H_{(s, t)}(R)$. There exist $C=\left[\begin{array}{ccc}x^{\prime} & 0 & 0 \\ y^{\prime} & z^{\prime} & t^{\prime} \\ 0 & 0 & u^{\prime}\end{array}\right] \in C\left(H_{(s, t)}(R)\right)$ and $U=\left[\begin{array}{lll}x & 0 & 0 \\ y & z & t \\ 0 & 0 & u\end{array}\right] \in U\left(H_{(s, t)}\right)(R)$ such that $A=U+C$. Then $x^{\prime} \in C(R)$ and $x \in U(R)$. So $a=x^{\prime}+x$ is a $C U$-decomposition of $a$.

We have proved that every uniquely nil clean ring is $C U$. There are $C U$ rings that are not uniquely nil clean.

Example 3.6. The ring $H_{(0,0)}(\mathbb{Z})$ is $C U$ but not uniquely nil clean.

Proof. By Theorem 3.5, $H_{(0,0)}(\mathbb{Z})$ is $C U$. Note that for $a \in \mathbb{Z}$ has a uniquely nil clean decomposition if and only if $a=0$ or $a=1$. Let $A=\left[\begin{array}{lll}a & 0 & 0 \\ c & a & e \\ 0 & 0 & a\end{array}\right] \in$ $H_{(0,0)}(R)$. Assume that $A$ has a uniquely nil clean decomposition. There exist unique $E^{2}=E=\left[\begin{array}{ccc}x & 0 & 0 \\ y & x & u \\ 0 & 0 & x\end{array}\right] \in H_{(0,0)}(R)$ and $N=\left[\begin{array}{lll}g & 0 & 0 \\ h & g & l \\ 0 & 0 & g\end{array}\right] \in N\left(H_{(0,0)}(R)\right.$ such that $A=E+N$. Then $A$ has a uniquely nil clean decomposition. This is not the case for each $a \in \mathbb{Z}$. Hence $H_{(0,0)}(\mathbb{Z})$ is not uniquely nil clean. 
Generalized matrix rings: Let $R$ be ring and $s$ a central element of $R$. The four tuple $\left[\begin{array}{ll}R & R \\ R & R\end{array}\right]$ becomes a ring denoted by $K_{s}(R)$ with addition defined componentwise and with multiplication defined in [5] by

$$
\left[\begin{array}{ll}
a_{1} & x_{1} \\
y_{1} & b_{1}
\end{array}\right]\left[\begin{array}{ll}
a_{2} & x_{2} \\
y_{2} & b_{2}
\end{array}\right]=\left[\begin{array}{cc}
a_{1} a_{2}+s x_{1} y_{2} & a_{1} x_{2}+x_{1} b_{2} \\
y_{1} a_{2}+b_{1} y_{2} & s y_{1} x_{2}+b_{1} b_{2}
\end{array}\right]
$$

Then $K_{S}(R)$ is called generalized matrix ring over $R$.

Lemma 3.7. Let $F$ be a field. Then

(1) $U\left(K_{0}(F)\right)=\left\{\left[\begin{array}{ll}a & b \\ c & d\end{array}\right] \in K_{0}(F) \mid a \neq 0, d \neq 0\right\}$.

(2) $C\left(K_{0}(F)\right)$ consists of all scalar matrices.

Proof. (1) Clear from [8, Lemma 3.1].

(2) It follows from [6, Lemma 1.1].

Theorem 3.8. Let $F$ be a field with $|F| \geq 3$. Then $K_{0}(F)$ is a CU ring.

Proof. Let $A=\left[\begin{array}{ll}a & b \\ c & d\end{array}\right]$ be any matrix. Since $|F| \geq 3$, we can find some $u \in F$ such that $a-u, d-u \in U(F)$. Let $C=\left[\begin{array}{ll}u & 0 \\ 0 & u\end{array}\right]$. Then $A-C \in U\left(K_{0}(F)\right)$.

Example 3.9. Let $F$ be a field with two elements. Then $K_{0}(F)$ is not $C U$.

Proof. Let $A=\left[\begin{array}{ll}1 & 1 \\ 0 & 0\end{array}\right] \in K_{0}(F)$. Suppose that $A$ has a $C U$-decomposition such that $A=C+U$ where $U=\left[\begin{array}{ll}x & y \\ z & t\end{array}\right]$ is invertible of which its main diagonal entries must be nonzero and $C=\left[\begin{array}{ll}c & 0 \\ 0 & c\end{array}\right]$ is central. $A=C+U$ implies $c+x=1$ and $c+t=0$. We complete the discussion by two cases :

Case I. $c=0 . t+c=0$ implies $t=0$. Invertibility of $U$ requires $t=1$. A contradiction.

Case II. $c=1$. Then $t=1$ and $x=0$. Again a contradiction. Hence $A$ does not have a $C U$-decomposition.

Acknowledgment. The authors would like to thank the referee for the valuable suggestions and comments. 


\section{References}

[1] H. Chen, Rings Related Stable Range Conditions, Series in Algebra 11, World Scientific, Hackensack, NJ, 2011.

[2] A. J. Diesl, Nil clean rings, J. Algebra, 383 (2013), 197-211.

[3] A. Harmanci, H. Chen and A. Özcan, Strongly nil *-clean rings, J. Algebra Comb. Discrete App. 4(2) (2017), 155-164.

[4] D. Khurana, G. Marks and A. Srivastava, On unit-central rings, Advances in ring theory, 205-212, Trends Math., Birkhauser-Springer Basel AG, Basel, 2010.

[5] P. A. Krylov, Isomorphism of generalized matrix rings, Algebra Logic, 47(4)(2008), 258-262.

[6] P. A. Krylov and A. A. Tuganbaev, Modules over formal matrix rings, J. Math. Sci. 171(2)(2010), 248-295.

[7] C. Li and Y. Zhou, On strongly *-clean rings, J. Algebra Appl., 10(6)(2011), 1363-1370.

[8] G. Tang, C. Li and Y. Zhou, Study of Morita contexts, Comm. Algebra 42(4)(2014), 1668-1681.

Department of Mathematics, Bilkent University

Ankara, Turkey

email : yosum@fen.bilkent.edu.tr

Department of Mathematics, Ankara University,

Ankara, Turkey

email : halici@ankara.edu.tr

Department of Mathematics, Hacettepe University,

Ankara, Turkey

email : harmanci@hacettepe.edu.tr

Department of Mathematics, Hangzhou Normal University,

Hangzhou, China

email : huanyinchen@aliyun.com 